\title{
Good intermediate-rank lattice rules based on the weighted star discrepancy
}

\author{
Vasile Sinescu and Stephen Joe \\ Department of Mathematics, University of Waikato, \\ Hamilton, New Zealand \\ *E-mails:vs27@math.waikato.ac.nz,stephenj@math.waikato.ac.nz
}

\begin{abstract}
We study the problem of constructing good intermediate-rank lattice rules in the sense of having a low weighted star discrepancy. The intermediate-rank rules considered here are obtained by "copying" rank-1 lattice rules. We show that such rules can be constructed using a component-by-component technique and prove that the bound for the weighted star discrepancy achieves the optimal convergence rate.

Keywords: Intermediate-rank lattice rules, weighted star discrepancy, component-by-component construction.
\end{abstract}

\section{Introduction}

Integrals over the $d$-dimensional unit cube given by

$$
I_{d}(f)=\int_{[0,1]^{d}} f(\boldsymbol{x}) \mathrm{d} \boldsymbol{x}
$$

may be approximated by rank-1 lattice rules. These are quadrature rules defined by

$$
\frac{1}{n} \sum_{k=0}^{n-1} f\left(\left\{\frac{k \boldsymbol{z}}{n}\right\}\right) .
$$

Here, $\boldsymbol{z} \in \mathbb{Z}^{d}$ is the generating vector having all the components conveniently assumed to be relatively prime with $n$, while the braces around the vector indicate that we take the fractional part of each component of the vector.

In general terms, the "rank" of a lattice rule represents the minimum number of generating vectors required to produce the quadrature points. For $d$-dimensional integrals, lattice rules may have rank up to $d$. Further 
details on the definition and the representation of lattice rules can be found in [13] and [14].

In some practical applications, the first variables are the most important. Hence, it seems natural to consider lattice rules obtained by "copying" rank-1 lattice rules. If $\ell \geq 1$ is an integer satisfying $\operatorname{gcd}(\ell, n)=1$ and $r$ is a fixed integer taken from the set $\{0,1, \ldots, d\}$, then we can define the following lattice rule:

$$
Q_{N, d}(f)=\frac{1}{\ell^{r} n} \sum_{m_{r}=0}^{\ell-1} \ldots \sum_{m_{1}=0}^{\ell-1} \sum_{k=0}^{n-1} f\left(\left\{\frac{k \boldsymbol{z}}{n}+\frac{\left(m_{1}, \ldots, m_{r}, 0, \ldots, 0\right)}{\ell}\right\}\right) .
$$

For $r \geq 1$, this lattice rule is a rank- $r$ lattice rule or "intermediate-rank lattice rule". Let's remark that the lattice rule (2) has $N=\ell^{r} n$ distinct points and is obtained by copying the rank-1 lattice rule (1) $\ell$ times in each of the first $r$ dimensions. It is easy to observe that when $r=0$ or $\ell=1$, the lattice rule (2) is reduced to the rank-1 lattice rule (1).

Such intermediate-rank lattice rules have been previously studied in [5], [7], and [13]. Here, in order to construct these intermediate-rank lattice rules, we employ the "weighted star discrepancy" as a measure of "goodness". An unweighted star discrepancy (corresponding to an $L_{\infty}$ maximum error) has been previously used in [3] and in more general works such as [10] or [13], while the weighted star discrepancy has been used in [1], [4], and $[12]$.

\section{Bounds for the weighted star discrepancy}

Let's observe first that the quadrature points of the lattice rule (2) can be rewritten as:

$$
\left\{\frac{k \boldsymbol{z}}{n}+\frac{\left(m_{1}, \ldots, m_{r}, 0, \ldots, 0\right)}{\ell}\right\}=\frac{\boldsymbol{y}_{t}}{N}
$$

where $\boldsymbol{y}_{t} / N, 0 \leq t \leq N-1$, are in $[0,1)^{d}$. Of course, these points are a reordering of the $N$-points of the rank- $r$ lattice rule defined by (2). Hence the lattice rule (2) may be rewritten as

$$
Q_{N, d}(f)=\frac{1}{N} \sum_{t=0}^{N-1} f\left(\frac{\boldsymbol{y}_{t}}{N}\right) .
$$

In order to introduce the weighted star discrepancy, let the set of quadrature points $\left\{\boldsymbol{y}_{t} / N, 0 \leq t \leq N-1\right\}$ be denoted by $P_{N}$. Then the star discrepancy 
of $P_{N}$ is defined by

$$
D_{N}^{*}\left(P_{N}\right):=\sup _{\boldsymbol{x} \in[0,1)^{d}}\left|\operatorname{discr}\left(\boldsymbol{x}, P_{N}\right)\right|,
$$

where $\operatorname{discr}\left(\boldsymbol{x}, P_{N}\right)$ is the local discrepancy given by

$$
\operatorname{discr}\left(\boldsymbol{x}, P_{N}\right):=\frac{A\left([\mathbf{0}, \boldsymbol{x}), P_{N}\right)}{N}-\prod_{j=1}^{d} x_{j} .
$$

Here $A\left([\mathbf{0}, \boldsymbol{x}), P_{N}\right)$ represents the counting function, namely the number of points in $P_{N}$ which lie in $[\mathbf{0}, \boldsymbol{x})$ with $\boldsymbol{x}=\left(x_{1}, x_{2}, \ldots, x_{d}\right)$. The star discrepancy gives a measure of the uniformity of the distribution of the quadrature points.

Let now $\mathfrak{u}$ be an arbitrary subset of $\mathcal{D}:=\{1,2, \ldots, d-1, d\}$ and denote its cardinality by $|\mathfrak{u}|$. For the vector $\boldsymbol{x} \in[0,1]^{d}$, let $\boldsymbol{x}_{\mathfrak{u}}$ denote the vector from $[0,1]^{|\mathfrak{u}|}$ containing the components of $\boldsymbol{x}$ whose indices belong to $\mathfrak{u}$. By $\left(\boldsymbol{x}_{\mathfrak{u}}, \mathbf{1}\right)$ we mean the vector from $[0,1]^{d}$ whose $j$-th component is $x_{j}$ if $j \in \mathfrak{u}$ and 1 if $j \notin \mathfrak{u}$. Now let us introduce a set of non-increasing positive weights $\left\{\gamma_{j}\right\}_{j=1}^{\infty}$ which describes the decreasing importance of the successive coordinates $x_{j}$ and set

$$
\gamma_{\mathfrak{u}}=\prod_{j \in \mathfrak{u}} \gamma_{j}
$$

From Zaremba's identity (see for instance [15] or [16]) and by applying Hölder's inequality for integrals and sums, we obtain

$$
\begin{aligned}
\left|Q_{N, d}(f)-I_{d}(f)\right| & \leq\left(\sum_{\mathfrak{u} \subseteq \mathcal{D}} \sup _{\boldsymbol{x}_{\mathfrak{u}} \in[0,1]^{|\mathfrak{u}|}} \gamma_{\mathfrak{u}}\left|\operatorname{discr}\left(\left(\boldsymbol{x}_{\mathfrak{u}}, \mathbf{1}\right), P_{N}\right)\right|\right) \\
& \times \sup _{\mathfrak{u} \subseteq \mathcal{D}} \gamma_{\mathfrak{u}}^{-1} \int_{[0,1]^{|\mathfrak{u}|}}\left|\frac{\partial^{|\mathfrak{u}|}}{\partial \boldsymbol{x}_{\mathfrak{u}}} f\left(\left(\boldsymbol{x}_{\mathfrak{u}}, \mathbf{1}\right)\right)\right| \mathrm{d} \boldsymbol{x}_{\mathfrak{u}} .
\end{aligned}
$$

Thus we can define a weighted star discrepancy $D_{N, \gamma}^{*}\left(P_{N}\right)$ by

$$
D_{N, \boldsymbol{\gamma}}^{*}\left(P_{N}\right):=\sum_{\mathfrak{u} \subseteq \mathcal{D}} \gamma_{\mathfrak{u}} \sup _{\boldsymbol{x}_{\mathfrak{u}} \in[0,1]^{|\mathfrak{u}|}}\left|\operatorname{discr}\left(\left(\boldsymbol{x}_{\mathfrak{u}}, \mathbf{1}\right), P_{N}\right)\right|
$$

From [10], we make use of Theorem 3.10 and Lemma 5.21, together with the arguments leading to Theorem 5.6, to obtain the following inequality:

$$
\sup _{\boldsymbol{x}_{\mathfrak{u}} \in[0,1]^{|\mathfrak{u}|}}\left|\operatorname{discr}\left(\left(\boldsymbol{x}_{\mathfrak{u}}, \mathbf{1}\right), P_{N}\right)\right| \leq 1-(1-1 / N)^{|\mathfrak{u}|}+\frac{R_{N}\left(P_{N}, \mathfrak{u}\right)}{2}
$$


where

$$
R_{N}\left(P_{N}, \mathfrak{u}\right)=\frac{1}{N} \sum_{t=0}^{N-1} \prod_{j \in \mathfrak{u}}\left(1+\sum_{-\frac{N}{2}<h \leq \frac{N}{2}}^{\prime} \frac{e^{2 \pi \mathrm{i} h y_{t, j} / N}}{|h|}\right)-1 .
$$

In the above $y_{t, j}$ is the $j$-th coordinate of $\boldsymbol{y}_{t}$, while the ${ }^{\prime}$ in the sum indicates we omit the $h=0$ term.

Let us mention here that from the general theory on lattice rules (for example, see [10] or [13]), it will follow that $R_{N}\left(P_{N}, \mathfrak{u}\right) \geq 0$ for any $\mathfrak{u} \subseteq \mathcal{D}$. From (3) and (4), we see that the general weighted star discrepancy satisfies the inequality

$$
D_{N, \gamma}^{*}\left(P_{N}\right) \leq \sum_{\mathfrak{u} \subseteq \mathcal{D}} \gamma_{\mathfrak{u}}\left(1-(1-1 / N)^{|\mathfrak{u}|}+\frac{R_{N}\left(P_{N}, \mathfrak{u}\right)}{2}\right) .
$$

Further bounds on the weighted star discrepancy may be obtained by making use of (5). If the weights $\gamma_{j}$ are summable, that is,

$$
\sum_{j=1}^{\infty} \gamma_{j}<\infty
$$

then from [4, Lemma 1], we obtain:

$$
\sum_{\mathfrak{u} \subseteq \mathcal{D}} \gamma_{\mathfrak{u}}\left(1-(1-1 / N)^{|\mathfrak{u}|}\right) \leq \frac{\max (1, \Gamma)}{N} \prod_{j=1}^{\infty}\left(1+\gamma_{j}\right) \leq \frac{\max (1, \Gamma)}{\ell^{r} n} e^{\sum_{j=1}^{\infty} \gamma_{j}},
$$

where

$$
\Gamma:=\sum_{j=1}^{\infty} \frac{\gamma_{j}}{1+\gamma_{j}}<\infty .
$$

The complete proof of this result may be found in [4]. Thus we obtain

$$
\sum_{\mathfrak{u} \subseteq \mathcal{D}} \gamma_{\mathfrak{u}}\left(1-(1-1 / N)^{|\mathfrak{u}|}\right)=O\left(n^{-1}\right)
$$

where the implied constant depends on $\ell, r$ and the weights.

We have from [4] that

$$
\sum_{\mathfrak{u} \subseteq \mathcal{D}} \gamma_{\mathfrak{u}} R_{N}\left(P_{N}, \mathfrak{u}\right)=\frac{1}{N} \sum_{t=0}^{N-1} \prod_{j=1}^{d}\left(\beta_{j}+\gamma_{j} \sum_{-\frac{N}{2}<h \leq \frac{N}{2}}^{\prime} \frac{e^{2 \pi \mathrm{i} h y_{t, j} / N}}{|h|}\right)-\prod_{j=1}^{d} \beta_{j},
$$

where $\beta_{j}=1+\gamma_{j}$. If we set

$$
e_{N, d}^{2}(\boldsymbol{z})=\sum_{\mathfrak{u} \subseteq \mathcal{D}} \gamma_{\mathfrak{u}} R_{N}\left(P_{N}, \mathfrak{u}\right)
$$


then we see that we have

$$
e_{N, d}^{2}(\boldsymbol{z})=\frac{1}{N} \sum_{t=0}^{N-1} \prod_{j=1}^{d}\left(\beta_{j}+\gamma_{j} \sum_{-\frac{N}{2}<h \leq \frac{N}{2}}^{\prime} \frac{e^{2 \pi \mathrm{i} h y_{t, j} / N}}{|h|}\right)-\prod_{j=1}^{d} \beta_{j} .
$$

Let's remark that the dependency on $\boldsymbol{z}$ in $e_{N, d}^{2}(\boldsymbol{z})$ makes sense as the vectors $\boldsymbol{y}_{t}$ actually depend on $\boldsymbol{z}$.

In research papers such as [2] or [5], it was proved that when $n$ is prime, the quantity (7) is identical to a quadrature error obtained from applying a rank-1 lattice rule to a certain integrand. Working with such a quadrature error simplifies in general the analysis of the problem and also has some computational advantages. Using the techniques from the mentioned papers, it is relatively easy to prove that

$$
e_{N, d}^{2}(\boldsymbol{z})=\frac{1}{n} \sum_{k=0}^{n-1} \prod_{j=1}^{d}\left(\beta_{j}+\tilde{\gamma}_{j} \sum_{-\frac{\tilde{N}_{j}}{2}<h \leq \frac{\tilde{N}_{j}}{2}}^{\prime} \frac{e^{2 \pi \mathrm{i} h k \hat{z}_{j} / n}}{|h|}\right)-\prod_{j=1}^{d} \beta_{j} .
$$

In the above, the following notations have been introduced:

$$
\tilde{\gamma}_{j}=\left\{\begin{array}{l}
\gamma_{j} / \ell, 1 \leq j \leq r \\
\gamma_{j}, \quad r+1 \leq j \leq d
\end{array}\right.
$$

Next,

$$
\tilde{N}_{j}= \begin{cases}N / \ell=\ell^{r-1} n, & 1 \leq j \leq r \\ N, & r+1 \leq j \leq d .\end{cases}
$$

Finally, $\hat{\boldsymbol{z}}=\left(\hat{z}_{1}, \hat{z}_{2}, \ldots, \hat{z}_{d}\right)$, with

$$
\hat{z}_{j}=\left\{\begin{array}{l}
\ell z_{j}, 1 \leq j \leq r \\
z_{j}, \quad r+1 \leq j \leq d .
\end{array}\right.
$$

Then by denoting

$$
f_{N}(\boldsymbol{x})=\prod_{j=1}^{d}\left(\beta_{j}+\tilde{\gamma}_{j} \sum_{-\frac{\tilde{N}_{j}}{2}<h \leq \frac{\tilde{N}_{j}}{2}}^{\prime} \frac{e^{2 \pi \mathrm{i} h x_{j}}}{|h|}\right)
$$

it is easy to observe that

$$
e_{N, d}^{2}(\boldsymbol{z})=\frac{1}{n} \sum_{k=0}^{n-1} f_{N}\left(\frac{k}{n} \hat{\boldsymbol{z}}\right)-\prod_{j=1}^{d} \beta_{j} .
$$

Now it is clear that $e_{N, d}^{2}(\boldsymbol{z})$ (which is based on a rank-r lattice rule with $N=\ell^{r} n$ points) can be obtained from applying a modified $n$-point rank-1 lattice rule to $f_{N}$. 
Next, we are looking to obtain a result for the mean of the quantities $e_{N, d}^{2}$. Such a result, together with (5) and (6), will allow us to deduce a certain bound for the weighted star discrepancy. This mean will be taken over all possible values of $\hat{\boldsymbol{z}}$. Because $\hat{\boldsymbol{z}}$ is known when $\boldsymbol{z}$ is known, the mean will be actually considered for all possible values for $\boldsymbol{z}$. Each component $z_{j}, 1 \leq j \leq d$, of the vector $\boldsymbol{z}$ can be taken from the set $\mathcal{Z}_{n}:=\{1,2, \ldots, n-$ $1\}$ because we only take the fractional part of each component of the vector. Thus, for prime $n$, the mean $M_{N, d, \gamma}$ is defined by

$$
M_{N, d, \gamma}:=\frac{1}{(n-1)^{d}} \sum_{\boldsymbol{z} \in \mathcal{Z}_{n}^{d}} e_{N, d}^{2}(\boldsymbol{z}) .
$$

An expression for $M_{N, d, \gamma}$ is given in the next theorem.

Theorem 2.1. If $n$ is prime, $\ell$ is a positive integer such that $\operatorname{gcd}(\ell, n)=1$ and $r$ is an integer chosen such that $1 \leq r \leq d$, then

$$
\begin{aligned}
M_{N, d, \gamma} & =\frac{1}{n} \prod_{j=1}^{d}\left(\beta_{j}+\tilde{\gamma}_{j} S_{\tilde{N}_{j}}\right) \\
& +\frac{n-1}{n} \prod_{j=1}^{d}\left(\beta_{j}-\frac{\tilde{\gamma}_{j}}{n-1}\left(S_{\tilde{N}_{j}}-S_{\tilde{N}_{j} / n}\right)\right)-\prod_{j=1}^{d} \beta_{j}
\end{aligned}
$$

where

$$
S_{n}=\sum_{-\frac{n}{2} \leq h<\frac{n}{2}}^{\prime} \frac{1}{|h|} \text {. }
$$

Proof. Using the definition of the mean and separating out the $k=0$ term in (8), we obtain:

$$
M_{N, d, \gamma}=\frac{1}{n} \prod_{j=1}^{d}\left(\beta_{j}+\tilde{\gamma}_{j} S_{\tilde{N}_{j}}\right)+\Theta_{N, \gamma}-\prod_{j=1}^{d} \beta_{j}
$$

where

$$
\begin{aligned}
\Theta_{N, \gamma} & =\frac{1}{n(n-1)^{d}} \sum_{\boldsymbol{z} \in \mathcal{Z}_{n}^{d}} \sum_{k=1}^{n-1} f_{N}\left(\frac{k}{n} \hat{z}\right) \\
& =\frac{1}{n} \sum_{k=1}^{n-1} \prod_{j=1}^{d}\left(\frac{1}{n-1} \sum_{z_{j}=1}^{n-1}\left(\beta_{j}+\tilde{\gamma}_{j} \sum_{-\frac{\tilde{N}_{j}}{2}<h \leq \frac{\tilde{N}_{j}}{2}}^{\prime} \frac{e^{2 \pi \mathrm{i} h k \hat{z}_{j} / n}}{|h|}\right)\right)
\end{aligned}
$$




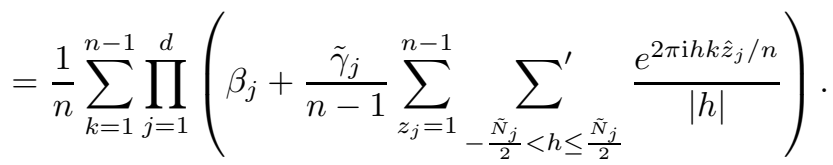

For $1 \leq k \leq n-1$ and for any $j \geq 1$, consider now

$$
T_{n}(k, j)=\sum_{z_{j}=1}^{n-1} \sum_{-\frac{\tilde{N}_{j}}{2}<h \leq \frac{\tilde{N}_{j}}{2}}^{\prime} \frac{e^{2 \pi \mathrm{i} h k \hat{z}_{j} / n}}{|h|} .
$$

By separating out the terms for which $h \equiv 0(\bmod n)$ and replacing $h$ by $n q$, we obtain

$$
\begin{aligned}
& T_{n}(k, j)=\sum_{z_{j}=1}^{n-1} \sum_{\substack{\tilde{N}_{j} \\
-\frac{\hat{N}_{j}}{2}<h \leq \frac{\tilde{N}_{j}}{2} \\
h \equiv 0(\bmod n)}}^{\prime} \frac{1}{|h|}+\sum_{z_{j}=1}^{n-1} \sum_{\substack{\tilde{N}_{j}<h \leq \frac{\tilde{N}_{j}}{2} \\
h \neq 0(\bmod n)}}^{\prime} \frac{e^{2 \pi \mathrm{i} h k \hat{z}_{j} / n}}{|h|} \\
& =\sum_{z_{j}=1}^{n-1} \sum_{-\frac{\tilde{N}_{j}}{2}<n q \leq \frac{\tilde{N}_{j}}{2}}^{\prime} \frac{1}{n|q|}+\sum_{\substack{\tilde{N}_{j}<h \leq \frac{\tilde{N}_{j}}{2} \\
h \neq 0(\bmod n)}}^{\prime} \frac{1}{|h|} \sum_{z_{j}=1}^{n-1}\left(e^{2 \pi \mathrm{i} h k / n}\right)^{\hat{z}_{j}} .
\end{aligned}
$$

If $\hat{z}_{j}=\ell z_{j}$, then

$$
\sum_{z_{j}=1}^{n-1}\left(e^{2 \pi \mathrm{i} h k / n}\right)^{\hat{z}_{j}}=\sum_{z_{j}=1}^{n-1}\left(e^{2 \pi \mathrm{i} h k \ell / n}\right)^{z_{j}} .
$$

Since $n$ is prime and $\operatorname{gcd}(\ell, n)=1$, then when $h \not \equiv 0(\bmod n)$, it follows that $h k \ell \not \equiv 0(\bmod n)$. It is then easy to check that

$$
\sum_{z_{j}=1}^{n-1}\left(e^{2 \pi \mathrm{i} h k \ell / n}\right)^{z_{j}}=-1 .
$$

When $\hat{z}_{j}=z_{j}$, the sum is the above with $\ell=1$ and has the same value of -1 . Replacing in the expression of $T_{n}(k, j)$ we obtain:

$$
T_{n}(k, j)=\frac{n-1}{n} S_{\tilde{N}_{j} / n}-\sum_{\substack{-\tilde{N}_{j} \\ h \neq h \leq \frac{\tilde{N}_{j}}{2}<(\bmod n)}}^{\prime} \frac{1}{|h|} .
$$

The last term of the sum may be written as:

$$
\sum_{\substack{\tilde{N}_{j} \\ h \neq h \leq \frac{\tilde{N}_{j}}{2} \\ h \neq 0(\bmod n)}}^{\prime} \frac{1}{|h|}=\sum_{-\frac{\tilde{N}_{j}}{2}<h \leq \frac{\tilde{N}_{j}}{2}}^{\prime} \frac{1}{|h|}-\sum_{-\frac{\tilde{N}_{j}}{2}<n q \leq \frac{\tilde{N}_{j}}{2}}^{\prime} \frac{1}{n|q|}
$$




$$
=S_{\tilde{N}_{j}}-\frac{1}{n} \sum_{-\frac{\tilde{N}_{j}}{2 n}<q \leq \frac{\tilde{N}_{j}}{2 n}}^{\prime} \frac{1}{|q|}=S_{\tilde{N}_{j}}-\frac{1}{n} S_{\tilde{N}_{j} / n}
$$

Thus we obtain:

$$
T_{n}(k, j)=\frac{n-1}{n} S_{\tilde{N}_{j} / n}-S_{\tilde{N}_{j}}+\frac{1}{n} S_{\tilde{N}_{j} / n}=S_{\tilde{N}_{j} / n}-S_{\tilde{N}_{j}} .
$$

Using now (12), we see that

$$
\Theta_{N, \gamma}=\frac{1}{n} \sum_{k=1}^{n-1} \prod_{j=1}^{d}\left(\beta_{j}+\frac{\tilde{\gamma}_{j}}{n-1}\left(S_{\tilde{N}_{j} / n}-S_{\tilde{N}_{j}}\right)\right),
$$

and by replacing in (10), we obtain the desired result.

From this theorem, we can deduce the following:

Corollary 2.1. If $n$ is a prime number, $\ell$ is a positive integer such that $\operatorname{gcd}(\ell, n)=1$ and $r$ satisfies $1 \leq r \leq d$, then there exists a $\boldsymbol{z} \in \mathcal{Z}_{n}^{d}$ such that

$$
e_{N, d}^{2}(\boldsymbol{z}) \leq \frac{1}{n} \prod_{j=1}^{d}\left(\beta_{j}+\tilde{\gamma}_{j} S_{\tilde{N}_{j}}\right) \leq \frac{1}{n} \prod_{j=1}^{d}\left(\beta_{j}+2 \tilde{\gamma}_{j} \ln \tilde{N}_{j}\right) .
$$

Proof. Since $\beta_{j}=1+\gamma_{j}$ for any $1 \leq j \leq d$, it will follow from $[9$, Lemmas 1 and 2] and the arguments used in [4] that

$$
\frac{n-1}{n} \prod_{j=1}^{d}\left(\beta_{j}-\frac{\tilde{\gamma}_{j}}{n-1}\left(S_{\tilde{N}_{j}}-S_{\tilde{N}_{j} / n}\right)\right)-\prod_{j=1}^{d} \beta_{j} \leq 0 .
$$

Using this in (9) together with the fact that $S_{\tilde{N}_{j}} \leq 2 \ln \tilde{N}_{j}$ for any $\tilde{N}_{j} \geq 2$ (see also [4] and [9]), we obtain

$$
M_{N, d, \gamma} \leq \frac{1}{n} \prod_{j=1}^{d}\left(\beta_{j}+\tilde{\gamma}_{j} S_{\tilde{N}_{j}}\right) \leq \frac{1}{n} \prod_{j=1}^{d}\left(\beta_{j}+2 \tilde{\gamma}_{j} \ln \tilde{N}_{j}\right)
$$

Clearly there must be a vector $z \in \mathcal{Z}_{n}^{d}$ such that

$$
e_{N, d}^{2}(\boldsymbol{z}) \leq M_{N, d, \gamma}
$$

This, together with the previous inequalities completes the proof.

From (5), (6) and Corollary 2.1, it follows that there exists a generating vector $\boldsymbol{z}$ such that

$$
D_{N, \gamma}^{*}(\boldsymbol{z}) \leq O\left(n^{-1}\right)+\frac{1}{2 n} \prod_{j=1}^{d}\left(\beta_{j}+2 \tilde{\gamma}_{j} \ln \tilde{N}_{j}\right)
$$


with the implied constant depending on $\ell, r$ and the weights, but independent of the dimension. As the above bound has a $\ln n$ dependency, it would appear that the weighted star discrepancy has the order of magnitude of $O\left(n^{-1}(\ln n)^{d}\right)$, a result which is widely believed to be the best possible in an unweighted setting (see [8] or [10] for details). However, in our case, under the assumption that the weights are summable, it follows from [1, Lemma 3] or [4, Lemma 2] that there exists a generating vector $\boldsymbol{z}$ such that the weighted star discrepancy achieves the strong tractability error bound

$$
D_{N, \boldsymbol{\gamma}}^{*}(\boldsymbol{z})=O\left(n^{-1+\delta}\right),
$$

for any $\delta>0$, where the implied constant depends on $\delta, \ell, r$ and the weights but is independent of $n$ and $d$.

\section{Component-By-Component Construction Of The Generating Vector}

In this section we show that intermediate-rank lattice rules of the form (2) that have good bounds for the weighted star discrepancy, can be obtained by making use of the so-named "component-by-component" (CBC) construction of the vector $\boldsymbol{z}$. This idea has been successfully used in several research papers such as [3], [4], [7], and [12] and is based on finding each component one at a time. The result is based on the following:

Theorem 3.1. Consider $n$ a prime number, $\ell$ a positive integer such that $\operatorname{gcd}(\ell, n)=1$ and $r$ chosen such that $1 \leq r \leq d$. Assume there exists a vector $\boldsymbol{z}$ in $\mathcal{Z}_{n}^{d}$ such that

$$
e_{N, d}^{2}(\boldsymbol{z}) \leq \frac{1}{n-1} \prod_{j=1}^{d}\left(\beta_{j}+\tilde{\gamma}_{j} S_{\tilde{N}_{j}}\right) .
$$

Then there exists a $z_{d+1} \in Z_{n}$ such that:

$$
e_{N, d+1}^{2}\left(\boldsymbol{z}, z_{d+1}\right) \leq \frac{1}{n-1} \prod_{j=1}^{d+1}\left(\beta_{j}+\tilde{\gamma}_{j} S_{\tilde{N}_{j}}\right) .
$$

Such a $z_{d+1}$ can be found by minimizing $e_{N, d+1}^{2}\left(\boldsymbol{z}, z_{d+1}\right)$ over $\mathcal{Z}_{n}$.

Proof. When we add a new component, we obtain from (8) that

$$
e_{N, d+1}^{2}\left(\boldsymbol{z}, z_{d+1}\right)=\frac{1}{n} \sum_{k=0}^{n-1} \prod_{j=1}^{d+1}\left(\beta_{j}+\tilde{\gamma}_{j} \sum_{-\frac{\tilde{N}_{j}}{2}<h \leq \frac{\tilde{N}_{j}}{2}}^{\prime} \frac{e^{2 \pi \mathrm{i} h k \hat{z}_{j} / n}}{|h|}\right)-\prod_{j=1}^{d+1} \beta_{j}
$$




$$
\begin{aligned}
= & \frac{1}{n} \sum_{k=0}^{n-1} \prod_{j=1}^{d}\left(\beta_{j}+\tilde{\gamma}_{j} \sum_{-\frac{\tilde{N}_{j}}{2}<h \leq \frac{\tilde{N}_{j}}{2}}^{\prime} \frac{e^{2 \pi \mathrm{i} h k \hat{z}_{j} / n}}{|h|}\right) \\
& \times\left(\beta_{d+1}+\tilde{\gamma}_{d+1} \sum_{-\frac{\tilde{N}_{d+1}}{2}<h \leq \frac{\tilde{N}_{d+1}}{2}}^{\prime} \frac{e^{2 \pi \mathrm{i} h k \hat{z}_{d+1} / n}}{|h|}\right)-\prod_{j=1}^{d+1} \beta_{j} .
\end{aligned}
$$

From (8) and by separating out the $k=0$ term in the above, we see that we can write

$$
\begin{aligned}
e_{N, d+1}^{2}\left(\boldsymbol{z}, z_{d+1}\right)= & \beta_{d+1} e_{N, d}^{2}(\boldsymbol{z})+\frac{\tilde{\gamma}_{d+1} S_{\tilde{N}_{d+1}}}{n} \prod_{j=1}^{d}\left(\beta_{j}+\tilde{\gamma}_{j} S_{\tilde{N}_{j}}\right) \\
& +\frac{\tilde{\gamma}_{d+1}}{n} \sum_{k=1}^{n-1} \prod_{j=1}^{d}\left(\beta_{j}+\tilde{\gamma}_{j} \sum_{-\frac{\tilde{N}_{j}}{2}<h \leq \frac{\tilde{N}_{j}}{2}}^{\prime} \frac{e^{2 \pi \mathrm{i} h k \hat{z}_{j} / n}}{|h|}\right) \\
& \times\left(\sum_{-\frac{\tilde{N}_{d+1}}{2}<h \leq \frac{\tilde{N}_{d+1}}{2}}^{\prime} \frac{e^{2 \pi \mathrm{i} h k \hat{z}_{d+1} / n}}{|h|}\right)
\end{aligned}
$$

We next average $e_{N, d+1}^{2}\left(\boldsymbol{z}, z_{d+1}\right)$ over all possible values of $z_{d+1} \in \mathcal{Z}_{n}$ and consider:

$$
\operatorname{Avg}\left(e_{N, d+1}^{2}\left(\boldsymbol{z}, z_{d+1}\right)\right)=\frac{1}{n-1} \sum_{z_{d+1}=1}^{n-1} e_{N, d+1}^{2}\left(\boldsymbol{z}, z_{d+1}\right)
$$

As the other terms that occur in the expression of the average are independent of $z_{d+1}$, we next focus on the quantity

$$
\frac{1}{n-1} \sum_{z_{d+1}=1}^{n-1} \sum_{-\frac{\tilde{N}_{d+1}}{2}<h \leq \frac{\tilde{N}_{d+1}}{2}}^{\prime} \frac{e^{2 \pi \mathrm{i} h k \hat{z}_{d+1} / n}}{|h|}=\frac{1}{n-1}\left(S_{\tilde{N}_{d+1} / n}-S_{\tilde{N}_{d+1}}\right),
$$

where we made use of (11) and (12). By replacing this equality in the expression of the average, we see that $\operatorname{Avg}\left(e_{N, d+1}^{2}\left(\boldsymbol{z}, z_{d+1}\right)\right)$ is given by:

$$
\begin{aligned}
& \beta_{d+1} e_{N, d}^{2}(\boldsymbol{z})+\frac{\tilde{\gamma}_{d+1} S_{\tilde{N}_{d+1}}}{n} \prod_{j=1}^{d}\left(\beta_{j}+\tilde{\gamma}_{j} S_{\tilde{N}_{j}}\right) \\
& +\frac{\tilde{\gamma}_{d+1}\left(S_{\tilde{N}_{d+1}}-S_{\tilde{N}_{d+1} / n}\right)}{n(n-1)} \times\left[-\sum_{k=1}^{n-1} \prod_{j=1}^{d}\left(\beta_{j}+\tilde{\gamma}_{j} \sum_{-\frac{\tilde{N}_{j}}{2}<h \leq \frac{\tilde{N}_{j}}{2}}^{\prime} \frac{e^{2 \pi \mathrm{i} h k \hat{z}_{j} / n}}{|h|}\right)\right] .
\end{aligned}
$$


Next,

$$
\begin{aligned}
& -\frac{1}{n} \sum_{k=1}^{n-1} \prod_{j=1}^{d}\left(\beta_{j}+\tilde{\gamma}_{j} \sum_{-\frac{\tilde{N}_{j}}{2}<h \leq \frac{\tilde{N}_{j}}{2}} \frac{e^{2 \pi \mathrm{i} h k \hat{z}_{j} / n}}{|h|}\right) \\
& =-\frac{1}{n} \sum_{k=0}^{n-1} \prod_{j=1}^{d}\left(\beta_{j}+\tilde{\gamma}_{j} \sum_{-\frac{\tilde{N}_{j}}{2}<h \leq \frac{\tilde{N}_{j}}{2}} \frac{e^{2 \pi \mathrm{i} h k \hat{z}_{j} / n}}{|h|}\right)+\frac{1}{n} \prod_{j=1}^{d}\left(\beta_{j}+\tilde{\gamma}_{j} S_{\tilde{N}_{j}}\right) \\
& =-e_{N, d}^{2}(\boldsymbol{z})-\prod_{j=1}^{d} \beta_{j}+\frac{1}{n} \prod_{j=1}^{d}\left(\beta_{j}+\tilde{\gamma}_{j} S_{\tilde{N}_{j}}\right) \leq \frac{1}{n} \prod_{j=1}^{d}\left(\beta_{j}+\tilde{\gamma}_{j} S_{\tilde{N}_{j}}\right) .
\end{aligned}
$$

In the last step we used $e_{N, d}^{2}(\boldsymbol{z}) \geq 0$, as $R_{N}\left(P_{N}, \mathfrak{u}\right) \geq 0$ for any $\mathfrak{u} \subseteq \mathcal{D}$ (see the previous section). Using also that $S_{\tilde{N}_{d+1}}-S_{\tilde{N}_{d+1} / n} \leq S_{\tilde{N}_{d+1}}$ and the hypothesis, we now obtain:

$$
\begin{aligned}
& \operatorname{Avg}\left(e_{N, d+1}^{2}\left(\boldsymbol{z}, z_{d+1}\right)\right) \\
\leq & \beta_{d+1} e_{N, d}^{2}(\boldsymbol{z})+\frac{\tilde{\gamma}_{d+1} S_{\tilde{N}_{d+1}}}{n} \prod_{j=1}^{d}\left(\beta_{j}+\tilde{\gamma}_{j} S_{\tilde{N}_{j}}\right) \\
& +\frac{\tilde{\gamma}_{d+1} S_{\tilde{N}_{d+1}}}{n(n-1)} \prod_{j=1}^{d}\left(\beta_{j}+\tilde{\gamma}_{j} S_{\tilde{N}_{j}}\right) \\
= & \beta_{d+1} e_{N, d}^{2}(\boldsymbol{z})+\frac{\tilde{\gamma}_{d+1} S_{\tilde{N}_{d+1}}}{n} \prod_{j=1}^{d}\left(\beta_{j}+\tilde{\gamma}_{j} S_{\tilde{N}_{j}}\right)\left(1+\frac{1}{n-1}\right) \\
\leq & \frac{\beta_{d+1}}{n-1} \prod_{j=1}^{d}\left(\beta_{j}+\tilde{\gamma}_{j} S_{\tilde{N}_{j}}\right)+\frac{\tilde{\gamma}_{d+1} S_{\tilde{N}_{d+1}}}{n-1} \prod_{j=1}^{d}\left(\beta_{j}+\tilde{\gamma}_{j} S_{\tilde{N}_{j}}\right) \\
= & \frac{1}{n-1} \prod_{j=1}^{d}\left(\beta_{j}+\tilde{\gamma}_{j} S_{\tilde{N}_{j}}\right)\left(\beta_{d+1}+\tilde{\gamma}_{d+1} S_{\tilde{N}_{d+1}}\right) .
\end{aligned}
$$

Clearly, the $z_{d+1} \in \mathcal{Z}_{n}$ chosen to minimize $e_{N, d+1}^{2}\left(\boldsymbol{z}, z_{d+1}\right)$ will satisfy

$$
e_{N, d+1}^{2}\left(\boldsymbol{z}, z_{d+1}\right) \leq \operatorname{Avg}\left(e_{N, d+1}^{2}\left(\boldsymbol{z}, z_{d+1}\right)\right) .
$$

This, together with the previous inequality completes the proof.

From this theorem we can deduce the following:

Corollary 3.1. Consider $n$ a prime number, $\ell$ a positive integer such that $\operatorname{gcd}(\ell, n)=1$ and $r$ chosen such that $1 \leq r \leq d$. Then for any $m=$ 
$1,2, \ldots, d$, there exists a $\boldsymbol{z} \in \mathcal{Z}_{n}^{m}$ such that

$$
e_{N, m}^{2}\left(z_{1}, z_{2}, \ldots, z_{m}\right) \leq \frac{1}{n-1} \prod_{j=1}^{m}\left(\beta_{j}+\tilde{\gamma}_{j} S_{\tilde{N}_{j}}\right),
$$

where

$e_{N, m}^{2}\left(z_{1}, z_{2}, \ldots, z_{m}\right)=\frac{1}{n} \sum_{k=0}^{n-1} \prod_{j=1}^{m}\left(\beta_{j}+\tilde{\gamma}_{j} \sum_{\substack{\tilde{N}_{j} \\-} h \leq \frac{\tilde{N}_{j}}{2}} \frac{e^{2 \pi \mathrm{i} h k \hat{z}_{j} / n}}{|h|}\right)-\prod_{j=1}^{m} \beta_{j}$.

We can set $z_{1}=1$ and for every $2 \leq m \leq d, z_{m}$ can be chosen by minimizing $e_{N, m}^{2}\left(z_{1}, z_{2}, \ldots, z_{m}\right)$ over the set $\mathcal{Z}_{n}$.

Proof. If $m=1$, then by expanding the expression of $e_{N, 1}^{2}\left(z_{1}\right)$ and using well-known results for geometrical series, we obtain that $e_{N, 1}^{2}\left(z_{1}\right)=0$ for any $z_{1} \in \mathcal{Z}_{n}$. The result then follows straight from Theorem 3.1.

\section{Component-by-component (CBC) algorithm}

The generating vector $\boldsymbol{z}=\left(z_{1}, z_{2}, \ldots, z_{d}\right)$ of a lattice rule (2) that satisfies the bound from Corollary 3.1 can be constructed as follows:

1. Set the value for the first component of the vector, say $z_{1}:=1$.

2. For $m=2,3, \ldots, d$, find $z_{m} \in \mathcal{Z}_{n}$ such that $e_{N, m}^{2}\left(z_{1}, z_{2}, \ldots, z_{m}\right)$ is minimized.

Clearly each $e_{N, m}^{2}\left(z_{1}, z_{2}, \ldots, z_{m}\right)$ can be evaluated in $O\left(n^{2} m\right)$ operations with a constant depending also on $\ell$ and $r$. This cost can be reduced to $O(\mathrm{~nm})$ by using asymptotic techniques as presented in [6] (see also [4, Appendix A]). Thus the total complexity of the algorithm will be $O\left(n^{2} d^{2}\right)$. This can be reduced to $O\left(n^{2} d\right)$ if we store the products during the construction at an extra expense of $O(n)$ storage. In fact, this order of complexity can be further reduced to $O(n d \log n)$ by making use of the fast CBC algorithm proposed by Nuyens and Cools in [11]. Their approach was based on minimizing a function of the form

$$
\frac{1}{n} \sum_{k=0}^{n-1} \prod_{j=1}^{d}\left(1+\gamma_{j} \omega\left(\left\{\frac{k z_{j}}{n}\right\}\right)\right)-1 .
$$

From (8), we know that $e_{N, d}^{2}(\boldsymbol{z})$ is obtained by applying a rank-1 lattice rule to a modified function, so the techniques used in [11] will also work here with some modifications. 


\section{References}

1. F.J. Hickernell and H. Niederreiter. (2003). The existence of good extensible rank-1 lattices, J. Complexity 19, pp. 286-300.

2. S. Joe. (1993). Bounds on the lattice rule criterion $R$, Math. Comp. 61, pp. 821-831.

3. S. Joe. (2004). Component by component construction of rank-1 lattice rules having $O\left(n^{-1}(\ln n)^{d}\right)$ star discrepancy, in Monte Carlo and Quasi-Monte Carlo Methods 2002, H. Niederreiter (Ed.), Springer, pp. 293-298.

4. S. Joe. (2006). Construction of good rank-1 lattice rules based on the weighted star discrepancy, in Monte Carlo and Quasi-Monte Carlo Methods 2004, H. Niederreiter and D. Talay (Eds), Springer, pp. 181-196.

5. S. Joe and S.A.R. Disney. (1993). Intermediate rank lattice rules for multidimensional integration, SIAM J. Numer. Anal. 30, pp. 569-582.

6. S. Joe and I.H. Sloan. (1992). On computing the lattice rule criterion $R$, Math. Comp. 59, pp. 557-568.

7. F. Kuo and S. Joe. (2003). Component-by-component construction of good intermediate-rank lattice rules, SIAM J. Numer. Anal. 41, pp. 1465-1486.

8. G. Larcher. (1987). A best lower bound for good lattice points, Monatsh. Math. 104, pp. $45-51$.

9. H. Niederreiter. (1978). Existence of good lattice points in the sense of Hlawka, Monatsh. Math. 86, pp. 203-219.

10. H. Niederreiter. (1992). Random number generation and quasi-Monte Carlo methods, SIAM, Philadelphia.

11. D. Nuyens and R. Cools. (2006). Fast algorithms for component-by-component construction of rank-1 lattice rules in shift-invariant reproducing kernel Hilbert spaces, Math. Comp. 75, pp. 903-920.

12. V. Sinescu and S. Joe. (2006). Good lattice rules based on the general weighted star discrepancy, Math. Comp., to appear.

13. I.H. Sloan and S. Joe. (1994). Lattice methods for multiple integration, Clarendon Press, Oxford.

14. I.H. Sloan and J.N. Lyness. (1989). The representation of lattice quadrature rules as multiple sums, Math. Comp. 52, pp. 81-94.

15. I.H. Sloan and H. Woźniakowski. (1998). When are quasi-Monte Carlo algorithms efficient for high dimensional integrals?, J. Complexity 14, pp. 1-33.

16. S.K. Zaremba. (1968). Some applications of multidimensional integration by parts, Ann. Polon. Math. 21, pp. 85-96. 